\title{
A Note on Water Transport Phenomenon by Homotopy Analysis Method
}

\author{
Twinkle Singh ${ }^{1}$ and R.K. Singh ${ }^{2}$ \\ ${ }^{1}$ Sardar Vallabhbhai national Institute of Technology, Surat-395007 \\ ${ }^{2}$ Uka Tarsadiya University, Bardoli-394 350
}

\begin{abstract}
In this paper, an analytic technique, named the Homotopy Analysis Method (HAM) has been applied for solving Richard's equation, which is converted into the Basic Burger's Equitation, which shows the well-known equations, to desire the behaviour of the infiltration of unsaturated zones in soil as a porous medium.
\end{abstract}

Keywords - Homotopy analysis method, Water transport phenomenon

\section{Introduction}

A number of non-linear phenomena in many branches of science and engineering such as physical, chemical, economical, environment, civil, water resources science, biological process are described by the interplay of reaction and diffusivity or by the interaction between convection and diffusion. One of the well known partial differential equations which given a wide variety of them is the Burger's Equation (BE) which provides the simplest non linear model of turbulence. Richards [11] derived a governing equation for water flow in soil based on continuum mechanics. In this model, the continuity equation was coupled with Darcy's law as a momentum equation. The following equation is known as the one dimensional form of Richards equation

$$
\frac{\partial C}{\partial T}=\frac{\partial}{\partial X}\left(D \frac{\partial C}{\partial X}-K\right)
$$

Where $C$ is unsaturated soil moisture content, $K$ is conductivity and $D$ is soil water diffusivity. Several methods are available for the estimation of such parameters, such as conductivity and water diffusivity [12]. Basically, there are three commonly used models: (i) Brook-Coreys model [1, 2] (ii) the van Genuchten model and (iii) the exponential model. The Brook-Corey model introduces a well-defined air-entry value that is associated with the largest pore-size, assuming complete wet ability Brooks- Corey model soils can be simplified to the following equations by some further considerations [3]:

$$
\begin{aligned}
& K(C)=K_{0} C^{k} \text { for } k \geq 1 \\
& D(C)=D_{0}(n+1) C^{n} \quad \text { for } n \geq 0
\end{aligned}
$$

where $K_{0}, D_{0}, n$ and $k$ are constants of representing soil properties such as pore-size distribution, particle shape etc. In these relations $C$ is called between 0 and 1 and the form of diffusivity is normalized so that

$$
\int_{0}^{1} D(C) d C=1 \quad \forall n
$$

There are several analytical and numerical solutions to Richards equation considering the Brook-Corey model. The choice $\mathrm{n}=0$ and $\mathrm{k}=0$ in equation (2) and (3) yields the classic Burger's Equations. Ayub et al [10] have been worked on porous plate with grade fluid with HAM.

The HAM contains the auxiliary parameter $\mathrm{h}$, which provides here a single way to adjust and control the convergence region of solution series for large values of $X$ and $T$. Other numerical methods are given with low degree of accuracy for large values of $X$ and $T$. Therefore, the HAM handles linear and non-linear problem without any assumption and restriction. In this study, a simplified Brook-Corey model (Equation (2) and (3)), was applied on Richard's equation [Equation (1)]. However two cases for conductivity exponents (with equal soil water diffusivity) and the solutions were tried. The general form of Burger's Equation in the order of $(\mathrm{n}, 1)$ is

$$
C_{T}+a\left(C^{n}\right)_{X}+b C_{X X}=0, \quad n \geq 1
$$

With the sake of convenience, with values of $n=2, a=1 / 2$, equation (5) reduces to 
$\frac{\partial C}{\partial T}+C \frac{\partial C}{\partial X}+b \frac{\partial^{2} C}{\partial X^{2}}=0 \quad$ where $b=-D$

With boundary conditions

$$
C(0, T)=C(1, T)=0 \quad T>0
$$

and the initial condition

$$
C(X, 0)=\pi X
$$

The equation (6) is the non-linear burger's equation in a water transport phenomenon together with boundary condition.

\section{Basic Idea of HAM}

We have apply the HAM [4], [5], [6], [7], [8] and [9] to the burgers' equation with boundary and initial conditions. The following differential equation has been considered

$$
N[\phi(X, T)]=0
$$

Where $N$ is a non linear operator for this problem $X$ and $T$ denote independent variables, $\phi(X, T)$ is an unknown function, for simplicity, we ignore here all boundary and initial conditions, which can be treated in the similar way. By means of the HAM, one first constructs zero-order deformation equation

$$
(1-\mathrm{p}) £\left[\varphi(\mathrm{X}, \mathrm{T} ; \mathrm{p})-C_{0}(X, T)\right]=\operatorname{phN}[\phi(\mathrm{X}, \mathrm{T} ; \mathrm{p})] \text {. }
$$

Where $£$ is an auxiliary linear operator, $C_{0}(X, T)$ is an initial guess, $h \neq 0$ is an auxiliary parameter and $p \in[0,1]$ is the embedding parameter. Obviously, when $p=0$ and $p=1$, it holds

$$
\varphi(\mathrm{X}, \mathrm{T} ; 0)=C_{0}(X, T) \quad \varphi(\mathrm{X}, \mathrm{T} ; 1)=\mathrm{C}(\mathrm{X}, \mathrm{T})
$$

respectively. The solution $\phi(\mathrm{X}, \mathrm{T} ; \mathrm{p})$ varies from the initial guess $C_{0}(\mathrm{X}, \mathrm{T})$ to the solution $\mathrm{C}(\mathrm{X}, \mathrm{T})$. Liao [4], [5], [6], [7], [8] and [9] expanded $\phi(X, T ; p)$ in Taylor's series about the embedding parameter

$$
\varphi(\mathrm{X}, \mathrm{T}, \mathrm{p})=C_{0}(\mathrm{X}, \mathrm{T})+\sum_{m=1}^{+\infty} C_{m}(X, T) p^{m}
$$

where

$$
C_{m}(X, T)=\frac{1}{m !} \frac{\partial^{m} \varphi(X, T ; p)}{\partial p^{m}} \quad \text { at } p=0
$$

The convergence of the series (8) depends upon the auxiliary parameter $h$. If it is convergent at $p=1$, one has

$$
\phi(X, T)=C_{0}(X, T)+\sum_{m=1}^{+\infty} C_{m}(X, T)
$$

Define the vectors

$$
\bar{C}_{m}=\left[C_{0}(X, T), C_{1}(X, T), \ldots \ldots, C_{n}(X, T)\right]
$$

Differentiating the zeroth-order deformation equation (6) $m$ times with respect to $p$ and then diving them by $m$ ! and finally setting $p=0$, we get the following $m^{\text {th }}$ order deformation equation

$$
£\left[C_{m}(X, T)-\chi_{m} C_{m-1}(X, T)\right]=h R_{m}\left(\bar{C}_{m-1}\right)
$$

where

$$
R_{m}\left(\bar{C}_{m-1}\right)=\frac{1}{(m-1) !} \frac{\partial^{m-1} N[\varphi(X, T ; p)]}{\partial p^{m-1}} \quad \text { at } p=0
$$

and

$$
\chi_{m}= \begin{cases}0, & m \leq 1 \\ 1, & m>1\end{cases}
$$


It should be emphasized that $C_{m}(X, T)$ for $m \geq 1$ is governed by the linear equation (10) with the linear boundary conditions that come from original problem, which can be easily solved by symbolic computation software such as maple \& mathematica.

\section{Analysis of the method by the HAM}

HAM is applying to equation (6) to illustrate the strength of the method and to establish exact solutions for this problem. We choose the linear operator

$$
£[C(X, T ; p)]=\frac{\partial C(X, T ; p)}{\partial T}
$$

We now define a non linear operator as

$$
N[C(X, T ; p)]=\frac{\partial C(X, T ; p)}{\partial T}+C(X, T ; p) \frac{\partial C(X, T ; p)}{\partial X}-v \frac{\partial^{2} C(X, T ; p)}{\partial X^{2}}
$$

Using above definition, we construct the zeroth-order deformation equation

$$
(1-p) £\left[C(X, T ; p)-C_{0}(X, T)\right]=p h N[C(X, T ; p)]
$$

For $p=0 \& p=1$, we can write

$$
\left\{\begin{array}{l}
C(X, T ; 0)=C_{0}(X, T)=C(X, 0) \\
C(X, T ; 1)=C(X, T)
\end{array}\right.
$$

Thus, we obtain the $m^{\text {th }}$ order deformation equations.

$$
£\left[C_{m}(X, T)-\chi_{m} C_{m-1}(X, T)\right]=h R_{m}\left(\bar{C}_{m-1}\right)
$$

where

$$
R_{m}\left(\bar{C}_{m-1}\right)=\frac{\partial C_{m-1}(X, T ; p)}{\partial T}+\sum_{n=0}^{m-1} C_{n}(X, T ; p) \frac{\partial C_{m-1-n}}{\partial X}-v \frac{\partial^{2} C_{m-1}(X, T ; p)}{\partial X^{2}}
$$

Now the solution of the $m^{\text {th }}$ order deformation equations (16) for $m \geq 1$ become

$$
C_{m}(X, T)=\chi_{m} C_{m-1}(X, T)+h £^{-1}\left[R_{m}\left(\bar{C}_{m-1}\right)\right]
$$

So a few terms of series solution are as follows:

$$
\begin{aligned}
& C_{0}(X, T)=n \pi X \\
& C_{1}(X, T)=n^{2} h T \pi X\left[1-n^{2} \frac{\pi X^{2}}{2}+U \pi\right] \\
& C_{2}(X, T)=\frac{1}{4} \pi h T\left[\begin{array}{l}
4\left(1+h+3 h \pi^{2}+v\right)\left(1-n^{2} \frac{\pi X^{2}}{2}\right)+ \\
\pi\left(4 v+h\left(T+4 v+2 \pi^{2}+v^{2}\right)+3 h T\left(1-n^{2} \frac{\pi X^{2}}{2}\right)(n \pi X)\right)
\end{array}\right.
\end{aligned}
$$

The exact solution of equation (5) with conditions (6) and (7) was given by Cole [1] as

$$
C(X, T)=\frac{2 \pi v \sum_{n=1}^{\infty} a_{n} e^{-n^{2} \pi^{2} v T} n^{2} \pi X}{a_{0}+\sum_{n=1}^{\infty} a_{n} e^{-n^{2} \pi^{2} v T}\left(1-\frac{n^{2} \pi X^{2}}{2}\right)}
$$

where 


$$
a_{0}=\int_{0}^{1} \exp \left[-(2 \pi v)^{-1}\left(1-\left(1-\frac{n^{2} \pi X^{2}}{2}\right)\right)\right] d X
$$

and

$$
a_{n}=2 \int_{0}^{1} \exp \left[-(2 \pi v)^{-1}\left(1-\left(1-\frac{n^{2} \pi X^{2}}{2}\right)\right)\right]\left(1-\frac{n^{2} \pi X^{2}}{2}\right) d X
$$

\section{Conclusion:}

In this paper, Homotopy analysis method has been developed for solving Richard's equation for all classed of $\mathrm{X}$ and various intervals of T. It is apparently seen that Homotopy analysis method is very powerful and efficient technique in finding analytical solutions for wide classes of nonlinear problems. In comparison with other method, this method gives accurate numerical solution.

\section{Reference:}

[1] Brook, R.H. and A. T. Corey, Hydraulic Properties of Porous Media, Hydrol Paper 3, Colorado State University, Fort Collins, 1964.

[2] Corey, A. T., Mechanics of Immiscible Fluids in Porous Media, Water Resources Publication, Highlands Ranch, CO, 1994, pp: 252.

[3] Davood, D. Ganji, M. Esmaeilpour and E. Moheseni, Application of the Homotopy Perturbation Method to Micropolor Flow in a Porous Channel, 2009.

[4] Liao, S.J., The proposed homotopy analysis technique for the solution of nonlinear problems. PhD. Thesis, Shanghai Jiao Tong University, 1992.

[5] Liao, S.J., An explicit, totally analytic approximation of Blasius viscous flow problems. Int. J. Nonlinear Mech., 1999, 34: 759-778.

[6] Liao, S.J., Beyond perturbation; Introduction to the Homotopy Analysis Method. Champan and Hall/CRC Press, Boca Raton, 2003a.

[7] Liao, S.J., On the analytic solution of magneto hydrodynamic flows of non-Newtonian fluids over a stretching sheet. $J$. Fluid $\begin{array}{llll}\text { Mech., } & 2003 b, & \text { 488:189-212 } & \text { DOI: }\end{array}$ http://journals.cambridge.org/action/displayAbstract? fromPage $=$ online\&aid $=164083$

[8] Liao, S.J.,On the homotopy analysis method for nonlinear problems. Applied Mathe. Comput., 147 (2004), pp. 499513.10.1016/S0096-3003(02)00790-7

[9] Liao, S.J., A new branch of solutions of boundary-layer flows over a permeable stretching plate. Int. J. Heat Mass Transfer 48 (2005) 25292539

[10] M. Ayub, A. Rasheed and T. Hayat.Exact flow of a third grade fluid past a porous plate using homotopy analysis method, November, Pages 2091-2103 International Journal of Engineering Science Volume 41, Issue 18

[11] Richards, L.A., Capillary Conduction of Liquids through Porous Medium, Physics, I: 318-333,1931.

[12] Russo, D., Determining Soil Water Properties by Parameter Estimation: On the Selection of a Model for the Hydraulic Properties, Water Resources Res., 1991, 24:453-459. 